\title{
A maximum motion technique for assessment of color vision defects
}

\author{
CHARLES A. COLUIN and AVI CHAUDHURI \\ McGill University, Montreal, Quebec, Canada
}

\begin{abstract}
Several luminance-matching methods, such as flicker fusion and the minimum motion technique (MMT), are capable of detecting certain forms of abnormal color vision. We present evidence that the heterochromatic fusion nystagmus (HFN) luminance matching technique can discriminate among normal trichromats, protanopes, and deuteranomals. The HFN luminance matching technique has the advantage that it provides a positive indication of isoluminance (maximization of motion) as opposed to the MMT and flicker fusion methods, which indicate isoluminance by the minimization of motion and flicker, respectively. We tested 16 normal trichromats, 6 protanopes, and 4 deuteranomals with the HFN technique. Results indicate that HFN is a useful tool for examining color vision. Because the HFN stimulus elicits reflexive eye movements (optokinetic nystagmus) that follow the apparent motion of the stimulus, HFN luminance matching will be particularly useful in animal and infant research, where other color vision tests are difficult to implement.
\end{abstract}

In a number of research areas, information on color vision defects is useful but difficult to obtain. Such is the case, for example, in developmental research and in animal vision science. With infant or animal subjects, most of the traditional clinical and psychophysical methods for evaluating adult humans' color vision are difficult to implement, and lengthy or complex procedures such as preferential looking and behavioral training must be used instead. Simpler and more efficient methods cannot be used, because animal and infant subjects lack the ability to understand complex tasks and to give active responses concerning their own perceptual states. Color vision assessment methods that rely on reflexive feedback are therefore very useful in research involving these subject groups (see, e.g., Anstis, Murasugi, \& Cavanagh, 1990; Logothesis \& Charles, 1990; Maurer, Lewis, Cavanagh, \& Anstis, 1989).

Anstis and Cavanagh's (1983; Anstis, Cavanagh, Maurer, \& Lewis, 1987) minimum motion technique (MMT) is perhaps the most widely known method of using reflexive feedback to assess color vision. Subjects are shown a two-color grating that appears to drift in one direction when the luminance ratio between the colors is greater than 1.0 and in the opposite direction when the ratio is less than 1.0. The stimulus elicits optokinetic nystagmus (OKN), which varies in amplitude and direction with the luminance ratio and is generally consistent with perceived direction of motion. The points at which $\mathrm{OKN}$ direction reversals take place can therefore be used to estimate when an isoluminance condition has been reached.

We thank Vasile Diaconu, Jocelyn Faubert, and Olga Overbury for help and advice. This research was supported by a grant from the Medical Research Council of Canada (MA-13127). Inquiries and requests for reprints should be sent to C. A. Collin, Psychology Department, McGill University, 1205 Dr. Penfield, Montreal, PQ, H3A 1B1 Canada (e-mail: charles@hebb.psych.mcgill.ca).
The isoluminant point obtained will depend on the subject's spectral sensitivity function. This function is known to differ between normal trichromats and individuals with various forms of red/green color blindness (see, e.g., Devos, Spileers, \& Arden, 1996; Kinnear, 1986; Verriest, 1971). With the MMT, one can therefore use OKN data in order to indirectly detect these anomalous color vision types (see, e.g., Maurer et al., 1989). This offers a significant advantage over earlier methods such as flicker fusion, which rely on subjects' reports of their perceptions. To date, the MMT has been effective in distinguishing between normal and red/green deficient color vision in infants (Maurer et al., 1989) and in determining isoluminance points in nonhuman species such as monkeys (Logothesis \& Charles, 1990) and guppies (Anstis et al., 1990).

Chaudhuri and Albright $(1990,1992)$ have developed another motion stimulus that can be used in obtaining isoluminance ratios. The principle advantage of this stimulus, called the heterochromatic fusion nystagmus (HFN) stimulus, is that the isoluminant point is based on a condition of maximum motion perception, unlike in the MMT, in which a minimization of perceived motion is taken as the isoluminant point. The HFN stimulus appears to move in one direction when two chromatic components are isoluminant (luminance ratio $\approx 1.0$ ) and in the opposite direction when they are nonisoluminant (luminance ratio $<1.0$ or $>$ $1.0)$. As with the MMT, OKN eye movements are elicited when subjects view the HFN stimulus. These are maximized, in conjunction with perceived motion, at the isoluminance condition. This means that the HFN technique provides a positive endpoint. The maximization of the change in polarity (i.e., of $\mathrm{OKN}$ eye movements and/or perceived motion) itself is the measure of the isoluminance point, as opposed to the absence of signal that is used to assess isoluminance in the MMT and flicker fusion techniques. 
Techniques using the HFN stimulus have several advantages over other luminance matching techniques. For instance, the HFN technique is easier to use than the MMT with monkeys that have not been specifically trained to track visual stimuli or provide feedback on their perceptual states (Chaudhuri \& Albright, 1992). Also the method is flexible. The size, shape, and spacing of the stimulus components can be varied widely without altering the isoluminance point obtained (Chaudhuri \& Albright, 1992). Although spatial frequency has been shown to affect isoluminance data (Cavanagh, MacLeod, \& Anstis, 1987; Cavanagh, Tyler, \& Favreau, 1984), the spatially broadband nature of the HFN components means that these effects are averaged out. This has the advantage of making the technique flexible, but it may also be that the technique could be more sensitive if its spatial frequency domain were limited.

The HFN stimulus also functions across a wide range of temporal parameters. Though the flicker rate is optimal at around $15 \mathrm{~Hz}$, faster rates provide valid (though more variable) data (Chaudhuri \& Albright, 1992). Furthermore, the velocity of the pattern is not constrained by the need for frame-to-frame correlation, a requirement which limits the velocities that can be used with the MMT. With the HFN stimulus, any velocity capable of driving OKN can be used (Chaudhuri \& Albright, 1992).

Although the HFN technique has been applied in basic research in order to find isoluminant pairs of colors (Chaudhuri \& Albright, 1990, 1992), its ability to distinguish between normal and abnormal color vision in humans has not been assessed. Luminance matching techniques in general (e.g., MMT and flicker fusion) have several limitations as color vision screening devices. For instance, they cannot reliably separate dichromats from abnormal trichromats (e.g., deuteranomals from deuteranopes or protanomals from protanopes) because of large overlaps in the spectral sensitivities of these populations (Cavonius \& Kammann, 1984; Devos et al., 1996; Verriest \& Uvijils, 1989). Also, because of the small or nonexistent input of the S-cone system to the luminance channel (see, e.g., Nakano \& Kaiser, 1992), they are incapable of detecting tritan-type deficits. But they are capable of discriminating among normal trichromacy, protantype deficits, and (with somewhat reduced sensitivity) deutan-type deficits (Cavonius \& Kammann, 1984; Devos et al., 1996; Verriest \& Uvijils, 1989). The HFN technique and these methods share the underlying principle of luminance matching, so we expect that it will share the limitations above. However, they should also share the capability of distinguishing among normal trichromacy, protan-type deficits, and deutan-type deficits.

We report here the results of a study on the use of HFN to distinguish among three groups: normal trichromats, deuteranomals, and protanopes. We have found that the HFN technique is capable of distinguishing between normal trichromats and protanopes with high sensitivity and between normal trichromats and deuteranomals with lower sensitivity. It should therefore prove useful in studies of color vision, especially when animal or infant subjects are involved. A preliminary report of these results has appeared in abstract form (Collin \& Chaudhuri, 1996).

\section{METHOD}

\section{Subjects}

A total of 26 individuals ( 6 protanopes, 4 deuteranomals, and 16 normal trichromats) drawn from a variety of student and work force populations participated in the experiment. Subjects were recruited by announcements in undergraduate classes or were referred by ophthalmologists and fellow researchers. All the color vision defects were congenital. The number of protanopes who were recruited was unusually high compared with the number of deuteranomals; but other researchers in our geographical area have reported similar distributions of subjects (J. Faubert, personal communication, October 10, 1997), and we suspect that the usual distribution of color vision types for European populations may not be accurate for the French Canadian population which predominates in our area. The fact that deuteranopes and protanomals were not tested was not considered critical, because luminance matching techniques are known to be insensitive for distinguishing dichromats from abnormal trichromats (Birch, 1991).

Mean group ages $( \pm S D$ ) for normal trichromats, protanopes, and deuteranomals were $25.4 \pm 04.4,39.7 \pm 19.7$, and $27.5 \pm 06.8$ years, respectively. Eight of the normal trichromats were female; all other participants were male. Normal trichromats volunteered; subjects with abnormal color vision were paid for participation.

\section{Materials}

A copy of American Optical's Hardy-Rand-Rittler pseudoisochromatic plate test (AO H-R-R plates), a Farnsworth-Munsell 100-Hue test (FM-100), and a Nagel anomaloscope were used to determine subjects' color vision classifications. The AO H-R-R and FM-100 were presented under a Macbeth Lamp, providing a standard Type $\mathrm{C}$ illuminant. The luminance and colorimetric values of screen phosphors were measured with a Minolta Chromameter CS-100.

\section{Stimuli}

The HFN stimulus was generated on a PC installed with a graphics co-processor (Number Nine Computer Corp., Cambridge, MA) and was presented on a Phillips 2182DC Fast Refresh 21-in. monitor. Each pixel subtended approximately $2.7^{\prime}$ of arc at a viewing distance of $57 \mathrm{~cm}$.

Sample sections of frames from the HFN stimulus are shown in Figure 1. The stimulus consists of a two-color random checker field interspersed with small randomly positioned black dots. The field scrolls horizontally at $7.5 \% \mathrm{sec}$, with the checkers and dots moving coherently (i.e., maintaining their relative positions). As this motion occurs, the checkers counterphase at $15 \mathrm{~Hz}$. That is, every $0.0667 \mathrm{sec}$, the pattern moves over by one checker's width and all the checkers of Color 1 (e.g., red) switch to Color 2 (e.g., green), and vice versa. The dots simply move with the pattern and do not change colors, remaining black throughout.

When the checker colors are nonisoluminant, their reversals produce luminance flicker, which masks the motion of the black dots. Furthermore, the timing of the reversals with the motion of the field produces a reverse-phi effect (Anstis, 1970; Anstis \& Rogers, 1975), so that the pattern resembles a flickering field of squares drifting in the direction opposite the actual direction of the pattern movement. The black dots can be seen; but their motion is obscured, and they do not appear to move coherently in any given direction.

When the checker colors are isoluminant, the masking flicker and reverse-phi disappear, and the checkers fuse into a single ho- 
a) Checker Colors Non-Isoluminant

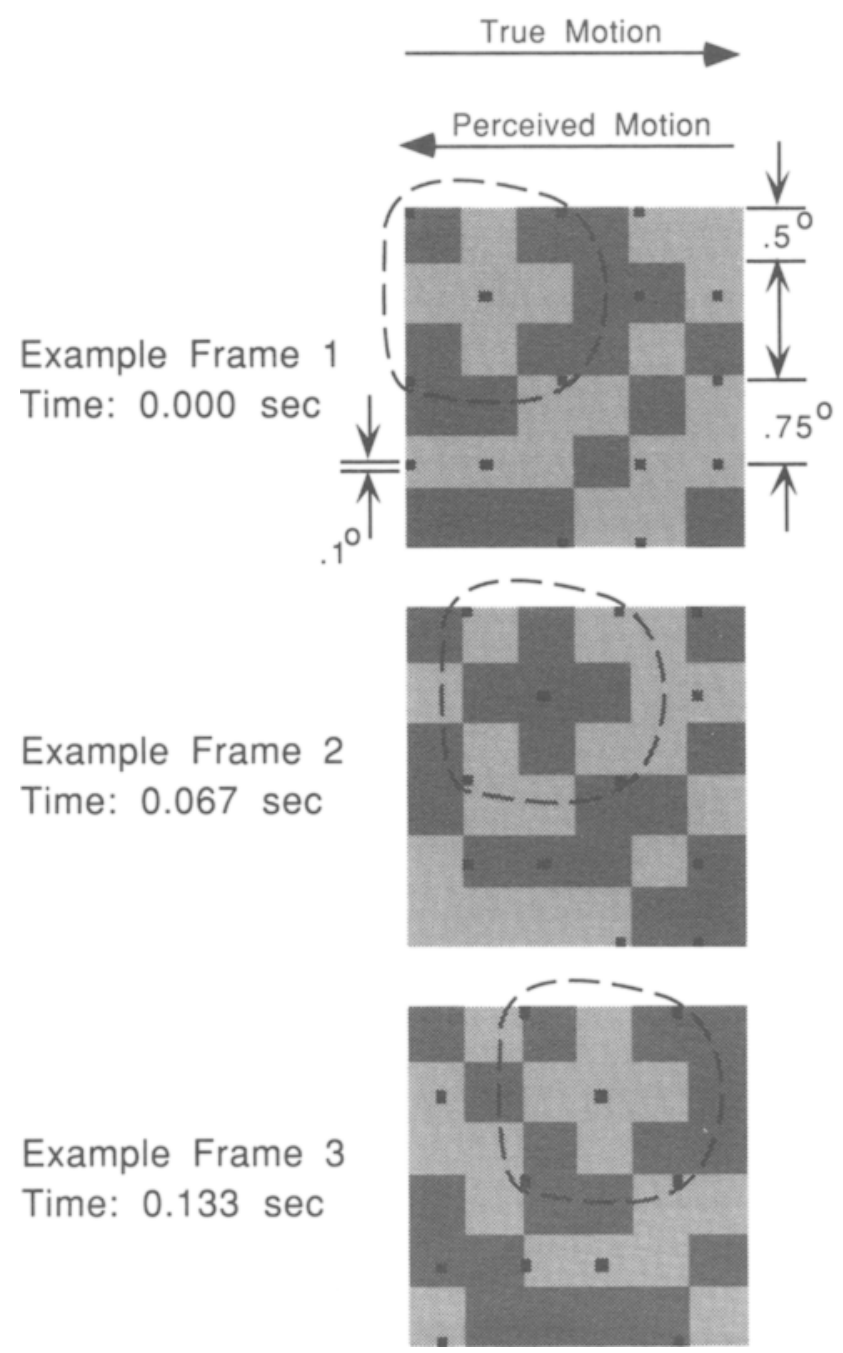

b) Checker Colors Isoluminant
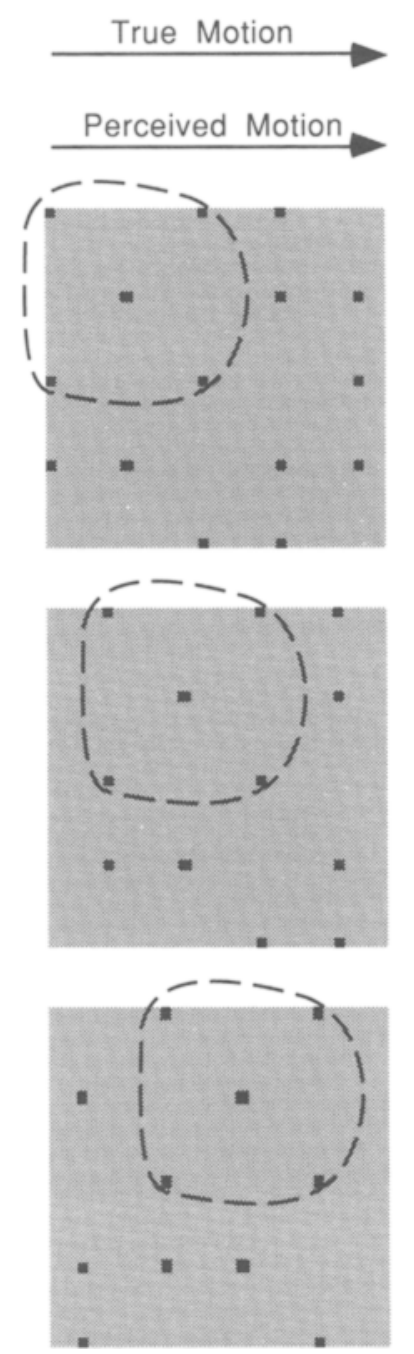

Figure 1. Sample sections of three frames of an HFN stimulus when checker colors are (a) nonisoluminant and (b) isoluminant. The pattern of checkers and dots moves coherently to the right in both examples. (a) $A$ crossshaped section of checkers (circled for emphasis) is moving rightward while switching colors across frames. The dots are unaffected and simply move with the pattern. When the checker colors are nonisoluminant, the movement of the black dots is masked by flicker and reverse phi. (b) When the checker colors are isoluminant, they fuse into an averaged background that exhibits minimal flicker. The black dots continue to move, however, as can be seen by examining the $x$-shaped configuration of dots (circled for emphasis). The perceived direction of motion is in the direction of the actual pattern scroll: Masking flicker and the reverse-phi effect have disappeared because of a lack of luminance contrast, and the motion of the black dots becomes salient.

mogenous background whose color is a mixture of the two checker colors. The black dots, no longer masked by flicker or reverse-phi, become visible and can be seen as moving in the true direction of the pattern's scroll. Thus, at isoluminance, the HFN resembles a static homogeneously colored background with a coherent pattern of black dots moving over it.

The parameters of the HFN stimuli used in this study were as follows. The checkers were $0.5^{\circ}$ squares randomly assigned one of two colors. The dots were $0.1^{\circ}$ black squares distributed over the random checker field in a grid pattern with positions separated by $0.75^{\circ}$. There was a $50 \%$ chance of a dot's appearing at any given position on this grid. The pattern was presented across the entire 21 in. screen, and it subtended approximately $36^{\circ} \times 28^{\circ}$ of visual ficld at the viewing distance of $57 \mathrm{~cm}$.

Two pairs of checker colors were used in this experiment: red on green, and red on blue. The red component (i.e., red phosphor) had a spectral energy function peaking around $610 \mathrm{~nm}$ (CIE coordinates: $\left.u^{\prime}=.413, v^{\prime}=.524\right)$ and the green had its peak at around $560 \mathrm{~nm}$ $\left(u^{\prime}=.125, v^{\prime}=.553\right)$. Figure 2 shows that these values correspond closely with the minimum and maximum of a function representing 


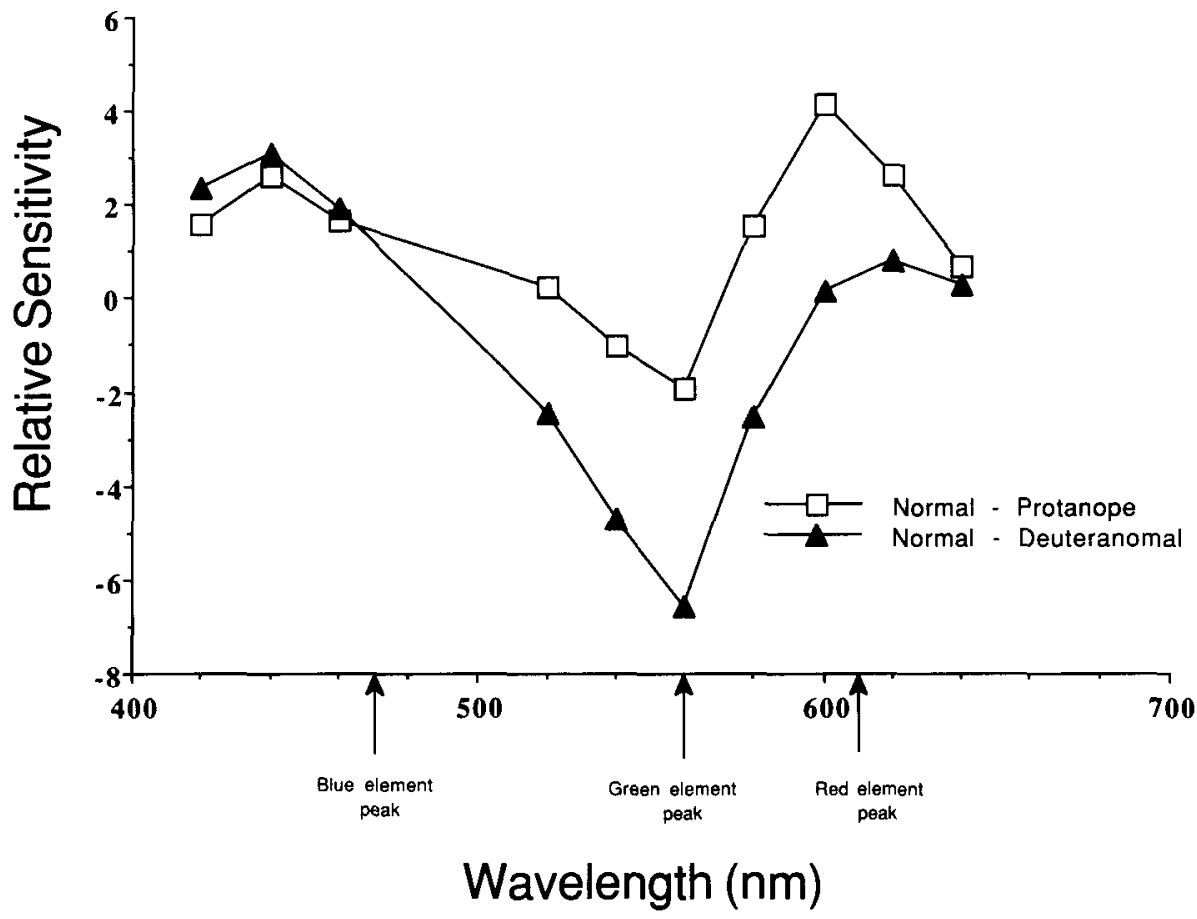

Figure 2. Relative differences in spectral sensitivity between normals and protanopes and between normals and deuteranomals. The figures were produced by subtracting the protanopic spectral sensitivity function from the normal trichromatic spectral sensitivity function (normal minus protanope) and by subtracting the deuteranomalous spectral sensitivity function from the normal function (normal minus deuteranomal).

the difference in spectral sensitivity between normals and protanopes. The function was generated by subtracting the protanopic spectral sensitivity function from the normal trichromatic function. The original spectral sensitivity functions were measured with a monochromator (J. Faubert, personal communication, October 10, 1997; see also Diaconu \& Faubert, 1997). At wavelengths where the difference function is positive, protanopes are less sensitive than normàls and vice versa. Because the dominant wavelength of the red components lies near the maximum of the function, they would appear much dimmer to protanopes than to normal trichromats at any given intensity. With the green, the situation is reversed, and the green phosphor would appear brighter to a protanope.

In the red-on-blue pair, the blue had a dominant wavelength of $470 \mathrm{~nm}\left(u^{\prime}=.168, v^{\prime}=.162\right)$. Figure 2 shows that this corresponds closely with the maximum of a function representing the difference in spectral sensitivity between normals and deuteranomals. The function was generated by subtracting the spectral sensitivity function for deuteranomals (Diaconu \& Faubert, 1997) from that for normals. Thus, deuteranomals will be less sensitive to wavelengths around the maximum of the function. This blue was again compared with the red described above

\section{Procedure}

Subjects were first tested and classified as to color vision type with AO H-R-R plates, the FM-100, and the Nagel anomaloscope, using standard procedures and criteria as outlined by the manufacturers (including the use of Macbeth Lamp illumination for the AO $\mathrm{H}-\mathrm{R}-\mathrm{R}$ and FM-100 tests). These three tests agreed in all cases as to the type of color blindness (protan vs. deutan) that the subject had, and they agreed quite well in terms of the degree of the disorder. Where tests disagreed significantly regarding the degree of the deficit (and this occurred for only 1 color anomalous subject), the anomaloscopy results were taken as correct. The results of these three tests for the color anomalous subjects are shown in Table 1. The subjects were subsequently evaluated with the HFN technique.

Each subject underwent 10 trials with the HFN procedure. In each trial, the subject was presented with a continuously scrolling HFN stimulus incorporating two color components. One of the components remained fixed in intensity while the subject adjusted the intensity of the other with a joystick. Subjects were asked to adjust the variable component until the impression of motion created by the black dot pattern was maximized (i.c., isoluminance was achieved). The intensity values at which they set the variable component were recorded automatically, and an average over 10 trials was taken. The direction of motion (left or right) was chosen randomly for each trial. At the beginning of each trial, the intensity of the test was randomly set at either its maximum or its minimum value.

For protanopes, the colors used in the pattern were red and green. The green was the standard component and was set to $9 \mathrm{~cd} / \mathrm{m}^{2}$. For deuteranomals, the colors used were red and blue. The blue was the standard component. Because of concerns about a mesopic shift in sensitivity toward the short wavelength end of the spectrum, the blue was placed at $10 \mathrm{~cd} / \mathrm{m}^{2}$, the maximum luminance that could be produced on our monitor while still maintaining the spectral characteristics noted above. Normal trichromats were tested under both these conditions.

\section{RESULTS}

Figure $3 \mathrm{a}$ shows the mean red/green luminance ratios at which protanopes and normal trichromats experienced isoluminance when viewing the HFN stimulus. It can be seen that the luminance ratio at which normal observers 
Table 1

Classifications of Color Anomalous Subjects by FM-100, AO H-R-R, and Nagel Anomaloscope Tests

\begin{tabular}{|c|c|c|c|c|c|}
\hline \multirow[b]{2}{*}{ Subject } & \multirow{2}{*}{$\begin{array}{c}\text { CV } \\
\text { Type* }\end{array}$} & \multicolumn{2}{|c|}{ FM-100 } & \multirow{2}{*}{$\begin{array}{c}\text { AO H-R-R } \\
\text { Classification } \\
\end{array}$} & \multirow{2}{*}{$\begin{array}{c}\text { Nagel Anomaloscope } \\
\text { Classification }\end{array}$} \\
\hline & & Error $^{\dagger}$ & Axis $\ddagger$ & & \\
\hline F.L. & $P$ & 104 & 65 (Protan) & Medium Protan & Dichromat-Protan \\
\hline D.S. & $\mathrm{P}$ & 128 & 65 (Protan) & Medium Protan & Dichromat-Protan \\
\hline A.M. & $\mathbf{P}$ & 132 & 62 (Protan) & Mild Protan & Dichromat-Protan \\
\hline D.G. & $P$ & 184 & 62 (Protan) & Medium Protan & Dichromat-Protan \\
\hline S.S. & $\mathbf{P}$ & 208 & 62 (Protan) & Strong Protan & Dichromat-Protan \\
\hline R.S. ${ }^{I}$ & $P$ & 312 & 62 (Protan) & Strong Protan & Dichromat-Protan \\
\hline P.B. & D & 148 & 60 (Deutan) & Medium Deutan & Abnormal Tri-Deutan \\
\hline R.S. ${ }^{2}$ & $\mathrm{D}$ & 168 & 56 (Deutan) & Medium Deutan & Abnormal Tri-Deutan \\
\hline J.N. & D & 176 & 60 (Deutan) & Medium Deutan & Abnormal Tri-Deutan \\
\hline N.P. & D & 168 & 60 (Deutan) & Medium Deutan & Abnormal Tri-Deutan \\
\hline
\end{tabular}

*Color vision type as determined by the three tests combined. P, protanope; D, deuteranomal. 'Error score for the FM-100: A score over 100 indicates abnormal color vision. \$Primary axis of error for the FM-100: A range of 62-70 indicates a protan-type deficit, whereas a range of 56-61 indicates a deutan-type deficit.

experienced isoluminance is close to unity $(M=1.04$, $S D=.21)$, while the same value for protanopes is quite high $(M=2.35, S D=.21)$. This difference is significant $(t=17.3, p<.001)$, and it agrees with the prediction that protanopes' lower sensitivity to long wavelengths would lead them to set the red component higher than would normals to achieve isoluminance with a green standard light.

The luminance ratios also agree reasonably well with the predicted ratios of 1.00 and 3.51 for normals and protanopes, respectively. These were calculated by first convolving the red and green phosphors' spectral energy functions with average spectral efficiency functions for normals and protanopes, and then making ratios of the integrals of the convolved functions. The fact that our empirical ratio for protanopes is somewhat lower than the theoretical ratio may be due to saturation effects. Our monitor's red gun produces a maximum of $30.5 \mathrm{~cd} / \mathrm{m}^{2}$, about 3.39 times the luminance of the $9-\mathrm{cd} / \mathrm{m}^{2}$ standard, so actually entering a ratio of 3.51 was not possible.

Figure $3 \mathrm{~b}$ shows the mean red/blue luminance ratios for deuteranomals and normal trichromats viewing the HFN stimulus. Here, an unexpected result occurs. Normal trichromats set the level of the variable red color's luminance far above that of the $10-\mathrm{cd} / \mathrm{m}^{2}$ blue standard, resulting in a luminance ratio greater than the expected unity value $\left(M=1.61, S D=.19 \mathrm{~cd} / \mathrm{m}^{2}\right)$. Deuteranomal subjects set the red luminance lower than did normals, which was as expected, but they too set the value above that of the standard $\left(M=1.29, S D=.19 \mathrm{~cd} / \mathrm{m}^{2}\right)$. The difference between the groups is significant $(t=3.3, p=$ .044 ) and is in the direction predicted. However, the absolute values are far above those expected.

On the basis of the spectral sensitivity curves of deuteranomals, it was predicted that they would place the luminance of the red variable lower than that of the blue standard, whereas normal trichromats would adjust the variable to match the standard. Our results suggest an artifact that elevated all responses by about $6 \mathrm{~cd} / \mathrm{m}^{2}$. Two possible explanations of this effect are based on the low luminance level of the test pattern: (1) there may have been a Purkinje shift in the spectral sensitivity of subjects, and (2) owing to the large spatial extent of the test pattern, rod intrusion may have altered the color perception of subjects. Also possible is an artifact due to phosphor crosstalk. These possibilities will be reviewed in more detail in the Discussion.

The empirically determined ratios also do not agree with the predicted ratios of 1.00 and 0.72 for normals and deuteranomals, respectively. These ratios, calculated as were those for the protanopes above, represent expectations based on the convolution of spectral energy functions from the monitor with the average spectral sensitivity for normals and deuteranomals. The empirically determined ratios come more into line with the theoretical ones if the ratio for normal subjects is scaled to unity and the same treatment is applied to the deuteranomal ratio, which then becomes 0.80 . If the ratios were indeed elevated by the postulated artifact, then scaling to unity shows that the relative differences between them agree with the predicted values.

The sensitivity and specificity of the HFN technique in detecting color vision anomalies was also assessed, though with the small number of subjects tested here these values must be regarded as quite tentative. For protanopes versus normals, the response ranges of the groups did not overlap, giving the test $100 \%$ sensitivity and specificity. The mean red/green luminance ratio at which protanopic subjects experienced isoluminance ranged from 2.18 to 2.71. For normal trichromats, the same range was .88 to 1.31. Thus, any criterion luminance ratio between 1.32 and 2.17 divided the groups completely.

For deuteranomals versus normals, there was some overlap of response ranges. Deuteranomals experienced isoluminance at red/blue luminance ratios ranging from 1.15 to 1.44 , whereas for normals the range was 1.44 to 1.83. The optimum criterion level was thus 1.44 (i.e., ratios of 1.44 and below resulted in classification as deuteranomal). With this criterion, sensitivity was $100 \%$ and 
a)

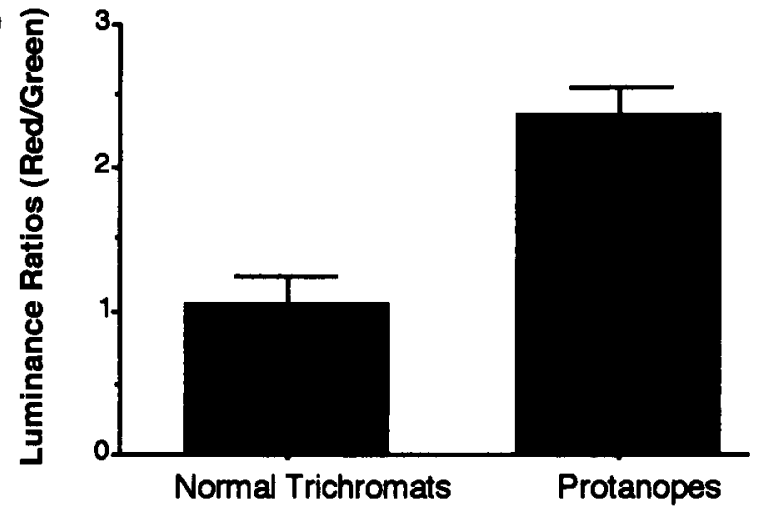

b)

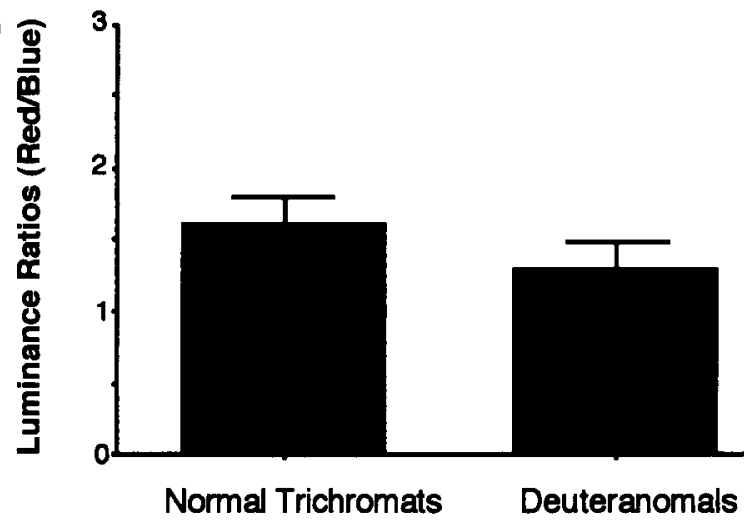

Figure 3. Mean luminance ratios for (a) normal trichromats and protanopes equating red variable with green standard checkers and (b) normal trichromats and deuteranomals equating red variable with blue standard checkers. The differences between groups in both cases are significant $(t=17.3, p<.001$ and $t=3.3$, $p=.044$, respectively). Error bars represent one standard error.

specificity was $87.5 \%$. That is, 2 of the 16 normal subjects would be misclassified as deuteranomals with this criterion.

To determine whether the classifications obtained using the HFN were concordant with those from the FM-100, kappa statistics were calculated (Bishop, Fienberg, \& Holland, 1975). The kappa statistic assesses whether two testing methods result in same classification of tested subjects. A kappa value above .7 is considered an indication of strong agreement. For the HFN, the classification criteria were the same as noted above. For the FM-100, the standard error score and primary axis criteria, as suggested by the manufacturers, were used as criteria for color vision classification. In the case of protanopes versus normals, the two tests agreed extremely well $(\kappa=1)$. In the case of deuteranomals versus normals, the tests also agreed strongly $(\kappa=.73, p<.01)$.

\section{DISCUSSION}

We have shown that the HFN technique is capable of distinguishing among protanopes, deuteranomals, and normal trichromats using keypress feedback. Though OKN measurements were not made here, previous work has shown that with the HFN technique, psychophysical data very closely parallel eye movement data. Also, casual observation indicates that the subjects (including color abnormal subjects) do indeed exhibit nystagmus at approximately the correct intervals during testing.

The sensitivity and specificity of the HFN technique seem to be high. These values appear to be lower for discrimination of deuteranomals from normals than for discrimination of protanopes from normals, but this is to be expected, because the difference in spectral sensitivity between normals and deuteranomals is much smaller than that between normals and protanopes. Many techniques for detecting color blindness show this pattern of greater sensitivity to protanopic deficits than to deuteranomalous deficits (Birch, 1991). The fact that the HFN results classify subjects in a manner very similar to those obtained with the FM-100 indicates that the technique has concordant validity with clinically used tests.

The unusually high response values associated with the blue-on-red HFN stimulus, whereby all subjects gave values approximately $6 \mathrm{~cd} / \mathrm{m}^{2}$ higher than was expected, require explanation. They may be the result of a mesopic shift in sensitivity. The blue gun of a computer monitor has limited energy output - the maximum luminance that could be produced for the blue checkers was $10 \mathrm{~cd} / \mathrm{m}^{2}-$ which is at the lower end of the photopic range. The results may therefore be explained by a shift in the spectral sensitivity curves of subjects toward the short-wavelength end of the spectrum as some rod photoreceptors become active in this range of luminance. This Purkinje shift would have the effect of making the blue checkers appear subjectively brighter while simultaneously making the red checkers appear dimmer (e.g., Vienot \& Chiron, 1992). We speculate that the effect is reduced with a green-on-red HFN stimulus because the peaks of the spectral content curves for the two guns are closer to one another and because there is a certain degree of overlap in the spectral energy functions of the two stimuli.

A second possible explanation for this result is rod intrusion (Stabell \& Stabell, 1976, 1979). Given the low luminance levels (lower end of photopic range) and the large spatial extent of the pattern, it is likely that rods in the extra-foveal retina were recruited, thereby changing color appearance. However, subjects generally reported paying attention to the central region of their vision, noting that the edges of the pattern continued to flicker when the central region was made to fuse. Rod intrusion effects are generally found in experiments in which subjects are asked to ignore the center of the field or are given an annular stimulus that masks the center of the field (Boynton, 1979).

The nature of the HFN stimulus makes it difficult to limit its spatial excursion to the fovea. However, we have developed a version of the stimulus that is limited to the central $10^{\circ}$ of visual field. This version also incorporates periodic bright adapting fields and a bright surround to 
keep the subject adapted to luminance levels that are unambiguously photopic. This should eliminate the concerns above in future research.

A third possible explanation for the elevated response levels in the red/blue condition concerns phosphor crosstalk. Color appearance might have been altered if the activation of one set of phosphors resulted in some activation in other sets of phosphors. However, our monitor has been carefully calibrated, and the spectral energy functions of the phosphors were measured extensively. These functions show no sign of cross-talk. Magnified visual inspection of the phosphors under various activation conditions corroborates this.

In conclusion, the HFN technique has been shown to be a useful tool for color vision assessment. Although it shares certain limitations with other luminance matching methods of color vision screening - for example, an inability to distinguish between dichromats and abnormal trichromats and detection of tritan-type deficits-both protanopes and deuteranomals can be distinguished from normal trichromats with the use of the HFN technique. The advantages of the HFN stimulus-positive endpoint and correlated $\mathrm{OKN}$ responses - provide this technique with a broad utility for color vision assessment that may be applicable when other tests are difficult to implement.

\section{REFERENCES}

ANSTIS, S. M. (1970). Phi movement as a subtractive process. Vision Research, 10, 1411-1430.

Anstis, S. M., \& CavanaGH, P. (1983) $)$. A minimum motion method for judging equiluminance. In J. D. Mollon \& L. T. Sharpe (Eds.), Color vision: Physiology and psychophysics (pp. 156-166). New York: Academic Press.

Anstis, S. M., Cavanagh, P., Maurer, D., \& Lewis, T. (1987). Optokinetic technique for measuring infants' response to color. Applied Optics, 27, 1510-1516.

anstis, S. M., Murasugi, C., \& Cavanagh, P. (1990). Optomotor test for wavelength sensitivity in guppyfish [Abstract]. Investigative Ophthalmology \& Visual Science, 31, 110.

ANSTIS, S. M., \& Rogers, B. J. (1975). Illusory reversal of visual depth and movement during changes of contrast. Vision Research, 15, 957-961.

BIRCH, J. (1991). Color vision tests: General classification. In J. R. Cronly-Dillon (Series Ed.) \& D. H. Foster (Vol. Ed.), Inherited and acquired colour vision deficiencies: Fundamental aspects and clinical studies. Vol. 7: Vision and visual dysfunction (pp. 215-234). Boston: CRC Press.

Bishop, Y. M., Fienberg, S. E., Holland, P. W. (1975). Discrete multivariate analysis: Theory and practice. Cambridge, MA: MIT Press.
Boynton, R. M. (1979). Human color vision. Washington, DC: Optical Society of America.

Cavanagh, P., MacLeod, D. I., \& Anstis, S. M. (1987). Equiluminance: Spatial and temporal factors and the contribution of bluesensitive cones. Journal of the Optical Society of America, 4, 1428-1438.

Cavanagh, P., Tyler, C. W., \& Favreau, O. E. (1984). Perceived velocity of moving chromatic gratings. Journal of the Optical Society of America, 1, 893-899.

Cavonius, C. R., \& Kammann, J. (1984). A clinical evaluation of the Oscar Color Vision Test. Documenta Ophthalmologica Proceedings Series, 39, 275-279.

Chaudhuri, A., \& Albright, T. D. (1990). A new technique for estimating chromatic isoluminance in humans and monkeys. Visual Neuroscience, 5, 605-608.

Chaudhuri, A., \& Albright, T. D. (1992). Heterochromatic fusion nystagmus: Its use in estimating chromatic equiluminance in humans and monkeys. Vision Research, 32, 1745-1759.

Collin, C., \& Chaudhuri, A. (1996). A maximum motion method for detection of red-green color deficiencies [Abstract]. Investigative Ophthalmology \& Visual Science, 37, 1062.

Devos, M., SPILEers, W., \& ARden, G. (1996). Colour contrast thresholds in congenital colour defectives. Vision Research, 36, 1055-1065.

DiACONU, V., \& FAUBERT, J. (1997). Evidence of red-green nonadditivity for deuteranomalous observers [Abstract]. Investigative Ophthalmology and Visual Science, 38 (4, Pt. 2), S894.

KINNEAR, P. R. (1986). Spectral sensitivity for observers with protanomalous, extreme protanomalous and protanopic colour vision. $O p h-$ thalmic \& Physiological Optics, 6, 197-200.

Logothesis, N. K., \& Charles, E. R. (1990). The minimum motion technique applied to determine isoluminance in psychophysical experiments with monkeys. Vision Research, 30, 829-838.

Maurer, D., Lewis, T., Cavanagh, P., \& Anstis, S. M. (1989). Testing the luminous efficiency of colors in babies. Investigative Ophthalmology \& Visual Science, 30, 297-303.

NAKANO, Y., \& KaISER, P. K. (1992). Color fusion and flicker fusion frequencies using tritanopic pairs. Vision Research, 32, 1417-1423.

Stabell, B., \& Stabell, U. (1976). Rod and cone contributions to peripheral colour vision. Vision Research, 16, 1099-1104

Stabell, B., \& STABell, U. (1979). Rod and cone contributions to change in hue with eccentricity. Vision Research, 19, 1121-1125.

VerRIEST, G. (1971). Les courbes spectrales photopiques d'efficacité lumineuse relative dans les déficiences congenitales de la vision des couleurs. Vision Research, 11, 1407-1434.

VERRIEst, G., \& UviJiLs, A. (1989). Results of the Oscar test in groups of normal and abnormal subjects. Documenta Ophthalmologica Proceedings Series, 52, 479-489.

VIENOT, F., \& ChIRON, A. (1992). Brightness matching and flicker photometric data obtained over the full mesopic range. Vision Research, 32, 533-540.

(Manuscript received July 16, 1997; revision accepted for publication November 19, 1997.) 